\title{
User Interface for Building a Secure and Comfortable Society - Proposals of New Light Curtain and Touch Switch -
}

\author{
Seiichi Serikawa, \\ ${ }^{a}$ Kyushu Institute of Technology, 1-1 Sensui, Tobata, Kitakyushu, Fukuoka, 804-8550, Japan \\ *Corresponding Author: serikawa@else.kyutech.ac.jp
}

\begin{abstract}
With the rapid decrease in the younger population, Japan has become an aging society in the recent years. Although more and more elder people are in need of care in their daily life, it is difficult for the work to be done by a relatively small workforce. Many elder people have to manage to take care of themselves with the help of automatic machines. Therefore, there is the very crucial task for engineers to make the user interface on these machines more understandable and easy to operate. In this speech, some examples of new user interfaces such as light curtain and touch switch are introduced and the advantages of these new inventions over the conventional designs are explained in detail.
\end{abstract}

